\title{
Die Cutting Formulation Process
}

National Cancer Institute

\section{Source}

National Cancer Institute. Die Cutting Formulation Process. NCI Thesaurus. Code C112969.

A process that forms a solid dosage unit by continuously cutting a uniform material into equally sized segments. 